\title{
REVISTA INGENIO
}

\section{Análisis de equilibrio de voltaje en un sistema de subtransmisión, mediante curvas QV-PV y análisis modal}

\section{Voltage stability analysis in a subtransmission system, using QV-PV curves and modal analysis}

\author{
Hólger Santillán | iD Universidad Politécnica Salesiana, Guayaquil, Ecuador \\ Rogger Peña|iD Instituto Superior Tecnológico Simón Bolívar, Guayaquil, Ecuador \\ Juan Morales|id Universidad Politécnica Salesiana, Guayaquil, Ecuador
}

HISTORIAL DEL ARTículo

Recepción: 15/06/2021

Aceptación: 07/09/2021

\section{PALABRAS Clave}

Inestabilidad de voltaje, potencias reactivas inductivas, voltajes, ángulos, PV, $\mathrm{QV}$, factores de participación, colapso de red.

\begin{abstract}
RESUMEN
El colapso de voltaje en una red eléctrica puede presentarse de diversas formas: como aumento de la carga, operatividad del máximo rendimiento de la máquina, puntos de generación muy alejados, potencias reactivas inductivas muy elevadas, entre otros, por lo que generan desequilibrio en su tensión y en los ángulos de los nodos en un sistema eléctrico. En la red de la provincia de Manabí se identificó, por medio de las curvas PV, que la barra de Chone de $69 \mathrm{kV}$ es la más crítica y, con las curvas QV, que la barra de Manta de $69 \mathrm{kV}$ es la de mayor criticidad, utilizando el software de DigSilent. Para obtener los factores de participación de los ramales y generadores, es necesario aumentar la carga a 0,1 Mvar para estimar la carga utilizando el análisis modal, en donde la subestación más crítica de los ramales es la de Quevedo, que tiene 0,8986 p. u. y en sus generadores la subestación es Montecristi, que llega a tener un valor de 0,346 p. u. en sus tensiones, utilizando Matlab para ejecutar el análisis. Este estudio es de gran importancia, ya que con esos valores de proyección se podrán observar los lugares en donde se presente una inestabilidad de voltaje y optimizar de diferentes formas la red, ya sea aumentando la generación, colocando bancos de capacitores, entre otros, en el punto donde se genere el colapso.
\end{abstract}

\begin{abstract}
The voltage collapse in an electrical network can occur in various ways such as: increased load, maximum performance of the machine, very remote generation points, very high inductive reactive powers, among others; therefore, they generate unbalance in their voltage and in the angles of the nodes in an electrical system. In the network of the Province of Manabí, it was identified by means of the PV curves that the Chone bar of $69 \mathrm{kV}$ is the most critical and with the QV curves that the Manta bar of $69 \mathrm{kV}$ is the most critical, using the software by DigSilent. To obtain the participation factors of the branches and generators, it is necessary to increase the load to 0.1 Mvar to estimate the load using modal analysis, where the most critical substation of the branches is the Quevedo substation, which has $0.8986 \mathrm{p}$. u. and in its generators the substation is Montecristi, which has a value of $0.346 \mathrm{p}$. u. on your stresses, using Matlab to run the analysis. This study is of great importance, since with these projection values it will be possible to observe the places where voltage instability is created and the network can be optimized in different ways, either by increasing the generation, by placing a capacitor bank, between others; at the point where the collapse is generated.
\end{abstract}

\section{INTRODUCCIÓN}

La inestabilidad del voltaje eléctrico, así como las variaciones en otras magnitudes físicas de un sistema eléctrico de potencia (SEP), como frecuencia y corriente, influyen directamente en el estándar de energía eléctrica proveída [1]. Es responsabilidad de las empresas de transmisión garantizar los niveles adecuados de dichas magnitudes y establecer normas y procedimientos para medir objetivamente la calidad de la energía que reciben los consumidores finales, reduciendo de esta forma sus efectos perjudiciales [2]. En Ecuador, esta responsabilidad recae en manos de Arconel y la regulación vigente está descrita en la resolución 053/18 [3]. 
La demanda y la generación eléctricas en Ecuador han crecido en los últimos años. Por ejemplo, del año 2019 al 2020 creció el 5,5\% y del año 2020 al 2021 crecerá el 5,2\%, que representa 5.091 MW, es decir, crecerá de acuerdo a lo proyectado en el Plan Maestro de Electricidad 2016-2025 [4]. Este aumento de la carga y complejidad del sistema eléctrico tiene un efecto en la estabilización del voltaje.

La inestabilidad de la tensión puede ser causada por perturbaciones eléctricas o electromecánicas; las empresas de transmisión tienen que ser capaces de identificar los diferentes tipos de problemas y proporcionar las soluciones correspondientes [5]. Un estudio de estabilización de diferencia de potencial permite determinar cuáles son los nodos críticos de la red y sus potenciales fallas con respecto al aumento de la carga [6]. El análisis de sensibilidad de la potencia activa y de la potencia reactiva de un SEP, ante las variaciones de voltaje que se realizan, utilizando las curvas PV (voltaje de potencia) y Qv (potencia reactiva) respectivamente, así como los datos aportados por un análisis modal del sistema, han probado ser herramientas suficientes para identificar los componentes críticos y evaluar el futuro desempeño del sistema en nuevos escenarios de carga. En este análisis se presentan los resultados de la inestabilidad de tensión utilizando curvas PV, QV y análisis modal del subsistema de subtransmisión de Portoviejo.

En el análisis de los resultados para la revisión sobre el estado del arte relacionado al desequilibrio de diferencia de potencial en una red eléctrica de potencia, se determinan las bases teóricas y el enfoque para el desarrollo del presente estudio y se relacionan las normas establecidas para evaluar el nivel de la energía entregada. En la sección III se describe la red eléctrica de la provincia de Manabí mediante un diagrama unifilar que ha sido modelado, parametrizado y simulado utilizando el software DigSilent Power Factory [7] y se describe la metodología utilizada en el análisis de sensibilidad utilizando las curvas $\mathrm{PV}, \mathrm{QV}$ y análisis modal. Además, se detalla el procedimiento utilizado en el análisis modal implementado en Matlab. En la sección IV se muestran y analizan los resultados centrados en el sistema de subtransmisión. Por último, se exterioriza el producto del análisis y se realizan las propuestas a fin de garantizar el futuro trabajo estable del sistema.

\section{MÉTODO}

SISTEMA ELÉCTRICO DE POTENCIA

\section{A. Aspectos generales}

Las redes de un sistema eléctrico de potencia están creadas para generar, transmitir y distribuir al usuario final voltaje, corriente y potencia según sus necesidades. En gran parte, los sistemas de generación se encuentran en zonas muy alejadas de la ciudad, por lo que es necesario elevar su nivel de tensión y evitar perturbaciones [8] para que la comercialización sea lo más económica posible hasta llegar a los centros de carga que son las subestaciones de distribución [9]. Es necesario contar con un software SCADA (el cual realiza inspección, revisión y adquisición de data) para poderlos controlar y ser capaz de mantener las redes en operaciones estables [10] y ante cualquier inestabilidad de voltaje alertar a los centros de operación y control para su respectiva inspección [11].

\section{B. Normas de calidad del servicio eléctrico en el Ecuador}

Las empresas de transmisión y subtransmisión están en la obligación de suministrar energía de calidad [12] a sus clientes. Arconel es la encargada de regular el servicio eléctrico y hacer cumplir las ordenanzas; una de las disposiciones sobre la calidad de voltaje está en su Resolución 053/18 que habla acerca de «la eficacia de funcionamiento de la repartición y mercadeo de energía eléctrica»[3], donde se especifica este tema de calidad de energía. Arconel divide las regulaciones en 3 aspectos muy importantes que son:

\section{Nivel de voltaje}

Conocer la intensidad del voltaje en un sistema de distribución hace posible determinar si los parámetros de calidad son aceptables, y de esta manera verificar la variación de voltaje en el suministro, la cual se determina de la siguiente forma [3]:

$\Delta V_{k}=\frac{V_{k}-V_{N}}{V_{N}} * 100[\%]$

Donde:

$\Delta V_{K}=$ Diferencial de la tensión de entrega respecto a la tensión nominal en el punto $k$.

$V_{k}=$ Tensión de entrega al punto $k$, definido como el promedio de las medidas registradas (por lo menos cada tres segundos) en un lapso de diez minutos.

$V_{N}=$ Tensión nominal en el punto $k$. 
Tabla 1. Límites de tensión aceptables por la Arconel 053/18

\begin{tabular}{lll}
\hline Nivel de voltaje & & Rango admisible \\
\hline Tensión alta-grupo 1 & $>138 \mathrm{kV}$ & $\pm 5.0 \%$ \\
Tensión alta-grupo 2 & $>40 \mathrm{kV} \mathrm{y} \leq 138 \mathrm{kV}$ & $\pm 5.0 \%$ \\
Tensión media & $>0,6 \mathrm{kV} \mathrm{y} \leq 40 \mathrm{kV}$ & $\pm 6.0 \%$ \\
Tensión baja & $\geq 0,6 \mathrm{kV}$ & $\pm 8.0 \%$ \\
\hline
\end{tabular}

\section{Límites de voltaje}

Es importante conocer cuáles son los rangos aceptables de voltaje en sus diferentes niveles de tensión; en la tabla 1 se observa los límites permisibles [3] (ver Tabla 1).

\section{Desequilibrio de voltaje}

Se puede analizar el punto donde existe un desequilibrio de voltaje y se obtiene de la siguiente fórmula [3]:

$$
\text { Desequilibrio de Voltaje }=\left|\frac{V^{-}}{V^{+}}\right| \times 100 \text { [\%] }
$$

Donde:

Componente de la secuencia negativa de tensión, definido como el producto medio de las medidas analizadas (por lo menos cada tres segundos) en un intervalo de diez minutos.

Componente de secuencia positiva de tensión, definido como el producto medio de las medidas analizadas (por lo menos cada tres segundos) en un intervalo de diez minutos.

\section{Tipos de desequilibrio de la tensión}

Se han descrito diferentes causas como generadoras de una inestabilidad de voltaje [13] que, al no controlarse, pueden ocasionar problemas graves en la red a los elementos, equipos y a los usuarios finales; por ello es necesario identificar cuáles de los diferentes tipos de inestabilidad de voltaje se están presentando en la red y llevarlos a los parámetros de calidad que exige la resolución de la Arconel 053/18 [3].

Algunos de los tipos de inestabilidad de voltaje más frecuente son:

\section{Aumento de la carga}

Cuando no existe un estudio de planificación adecuada del crecimiento de la demanda por ciudades, región y país, es difícil controlar la inestabilidad de voltaje [14], ya que la expansión territorial del ser humano va aumentando y con ello también la carga [15].

\section{Operabilidad del máximo rendimiento de la máquina}

Inicialmente, cualquier máquina en condiciones normales va a responder sin ningún problema ante la detección de una carga, pero a medida que va aumentando la carga se va perdiendo la eficiencia [16] ya que el rendimiento de la máquina llega a su máxima capacidad y entra a un estado de saturación [17].

\section{Puntos de generación muy alejados}

Cuando las fuentes de generación son muy alejadas, la impedancia de la línea hace disminuir el nivel de tensión en la que se encuentra [18], creando un bajo voltaje al final de la línea [19].

\section{Potencias reactivas inductivas muy elevadas}

En un sistema eléctrico de potencia, a medida que su carga va aumentando, también lo hará su potencia reactiva inductiva, por lo que se va a generar una inestabilidad tanto en voltaje como en ángulo [20].

\section{DigSilent}

DigSilent Power Factory es un software que ayuda a la simulación de cualquier sistema eléctrico para obtener los resultados necesarios y de esta manera desarrollar proyectos que ayuden a mejorar una red eléctrica en estudio.

Este software incorpora una librería muy grande para realizar diversos casos de estudio como: flujo de fuerza, estudios de fallas, estudio de mallas de baja tensión, mejoramiento de mallas de distribución, modelación dinámica, entre otros [21]. Es muy utilizado en las empresas eléctricas ecuatorianas como: Cenace y Transelectric porque dan un gran aporte al análisis de la red en estudio.

\section{RESULTADOS Y DISCUSIóN}

CURVAS PV-QV Y ANÁLISIS MODAL

\section{A. Red eléctrica de la provincia de Manabí}

El sistema eléctrico de la provincia de Manabí cuenta con 28 barras, 9 transformadores de tres devanados, 13 transformadores de dos devanados, 10 puntos de cargas y 4 puntos de bancos de transformadores trifásicos como se observa en la figura 1(ver Figura 1). 
Las 10 cargas que se encuentran acopladas a la red de estudio son: Severino, Chone, San Gregorio, Quevedo, Esquinas Lodana, Esquinas Portoviejo 3, Esquinas Portoviejo 1, Manta.

Con respecto a las 10 cargas conectadas a la red, se analiza la estabilidad en la tensión mediante las gráficas QV-PV, para estudiar el comportamiento dinámico de sus cargas activa y reactiva inductiva con respecto al análisis modal.

Para obtener las curvas, se comienza dando flujo al sistema eléctrico para observar cómo se está comportado la red en condiciones normales, con el reporte del BusBar se adquieren todos los datos de corriente, voltaje, frecuencia, entre otros. Después se escogen todas las barras que estén conectadas a una carga, para comparar su demanda con respecto al nivel de voltaje, y finalmente se obtienen las gráficas.

\section{B. Curva Pv}

El análisis de esta curva es muy importante, ya que con ello se observa el comportamiento del voltaje a medida que la potencia activa comienza a aumentar o disminuir en el sistema [22]. El análisis de las curvas Pv se interpreta desde su punto de colapso, a medida que su demanda de potencia activa va aumentando, su voltaje va a disminuir, por lo que la curva que se encuentre en la parte más inferior de los ejes de las «Y», que corresponde a las coordenadas de los voltajes, indicará cuál es la barra más crítica de la red (ver Figura 2) [23]. En la curva $\mathrm{PV}$, se indica que $\mathrm{P}$ es la cantidad de potencia activa que está entregando la barra y $\mathrm{v}$ es el cambio de la tensión a medida que la carga comienza a aumentar hasta alcanzar al punto de su máxima transferencia antes de llegar al colapso de tensión, como se observa en la figura 2 [24].

Con las 10 cargas anteriormente nombradas se obtienen las curvas PV por medio del software DigSilent. Cada barra tiene diferentes tipos de cargas, por lo que sus ángulos, voltajes y potencia va a variar, se obtuvo una curva por cada barra para analizar e identificar el punto de colapso en el bus más crítico del sistema de estudio [25].

\section{Curva Qv}

El análisis de este tipo de curva es muy importante ya que se observa la inestabilidad de voltaje en cada una de las barras por la falta del balance de la potencia reactiva que se genera en la red eléctrica [26]. La curva QV, tiene un aspecto en forma de $\mathrm{U}$, en donde el punto máximo de la curva se encuentra en su holgura, es decir, en la parte más baja de la curva y es ahí donde se encuentra la potencia reactiva más crítica con respecto al voltaje (ver Figura 3). Este tipo de inestabilidad se genera por el exceso de consumo o demanda de potencia reactiva inductiva, en donde se identificará, por medio de sus curvas, cuál es el punto de carga que está cerca del colapso por no balancear su demanda [27]. Por ejemplo, la figura 3 muestra que la gráfica más eficiente es la P1 ya que tiene una reserva para poder conectar muchas cargas más de potencia reactiva inductiva sin afectar la red. La curva P2 es el límite máximo de operación. En la curva P3 el sistema se encuentra en una inestabilidad de voltaje por falta de potencia reactiva capacitiva [28].

Con las mismas 10 cargas anteriormente nombradas se obtiene las curvas QV utilizando el software de DigSilent. Los ángulos, voltajes y potencia van a ser diferente ya que cada barra tendrá distintas demandas y con las curvas obtenidas se podrá analizar el punto más crítico que será la curva con la concavidad más alta [29].

\section{Análisis modal}

En el análisis modal se establecen las características como la frecuencia de resonancia, amortiguamiento, modo estado de excitación, entre otros. Los valores que sirven para satisfacer valores propios de la matriz.

El objetivo principal se basa en analizar las áreas más vulnerables a una inestabilidad de voltaje, que también se las denomina áreas débiles; que tienen un decaimiento de voltaje con respecto a su carga, que se analizará por medio de los resultados de los factores de participación [30].

Este análisis modal se basa en encontrar los valores propios que son los valores o modos del sistema que atañe de manera directa a la potencia reactiva introducida en el sistema junto a las tensiones en los nodos [31] o también llamados lambdas que se utilizan en la matriz jacobiana reducida en el procedimiento de Newton-Raphson, el cual consiste, principalmente, en analizar las variaciones en potencia reactiva y en variación de voltaje del sistema, siendo un método de ingeniería que se usa en el estudio de la inestabilidad de voltaje de una red eléctrica de fuerza, y mediante la cual se basa parte del análisis realizado en el presente estudio [32].

\section{Matriz jacobiana reducida}

Para obtener la matriz jacobiana reducida [33] es necesario dividir en cuatro partes la matriz del método de Newton-Raphson [34] y asumiendo que $\Delta \mathrm{P}=0$, se obtiene la siguiente expresión [27]:

$$
\left[\begin{array}{ll}
J_{11} & J_{12} \\
J_{21} & J_{22}
\end{array}\right]\left[\begin{array}{l}
\Delta \delta \\
\Delta V
\end{array}\right]=\left[\begin{array}{c}
0 \\
\Delta V
\end{array}\right]
$$

Resolviendo esta matriz se obtiene:

$$
\begin{aligned}
& J_{R}=\left[-J_{21} J_{11}{ }^{-1} J_{12}+J_{22}\right] \\
& J_{R} \Delta \mathrm{v}=\Delta \mathrm{Q}
\end{aligned}
$$


Figura 1. Red eléctrica de potencia de la provincia de Manabí

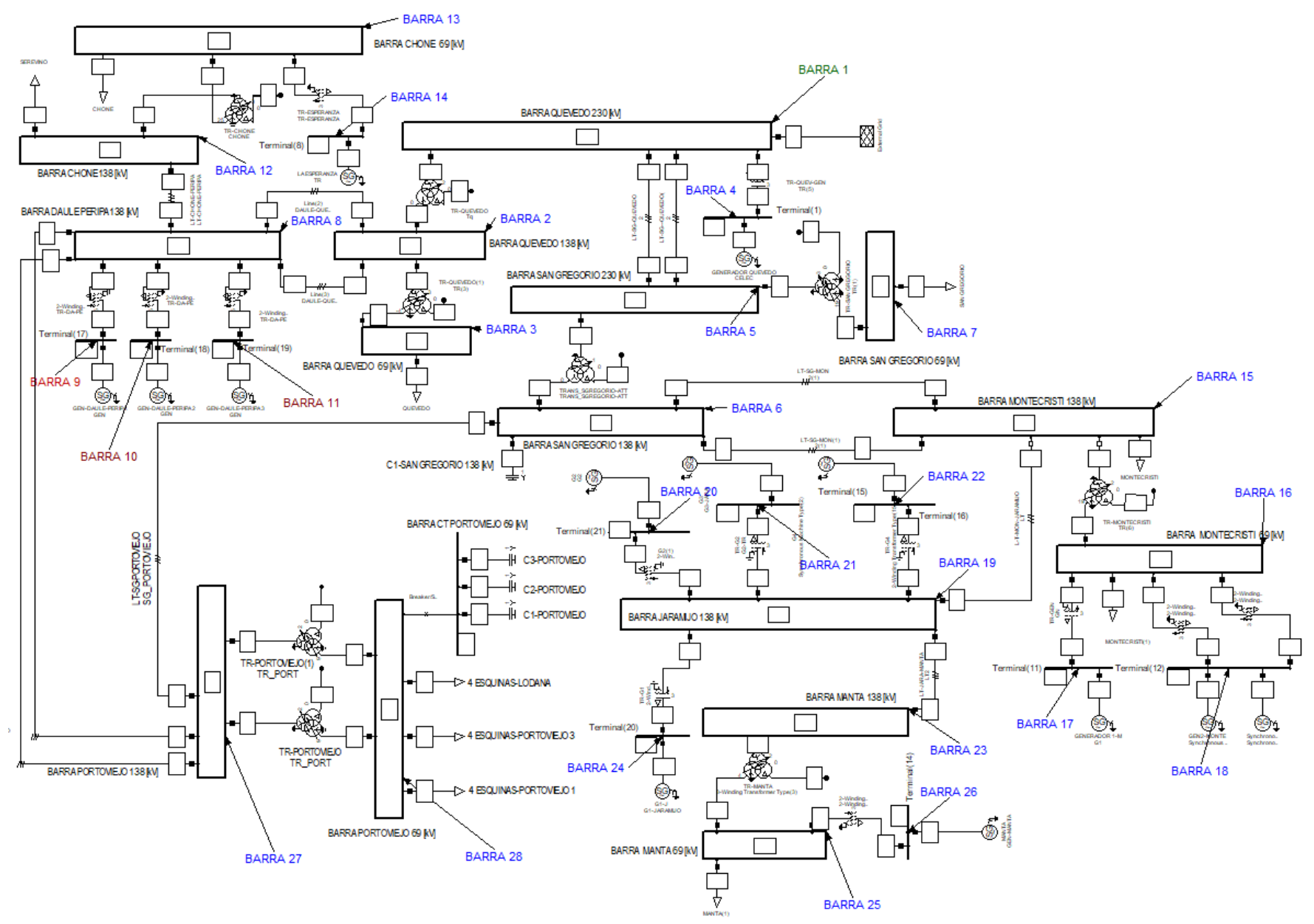

Figura 2. Áreas de estabilidad e inestabilidad de la curva PV

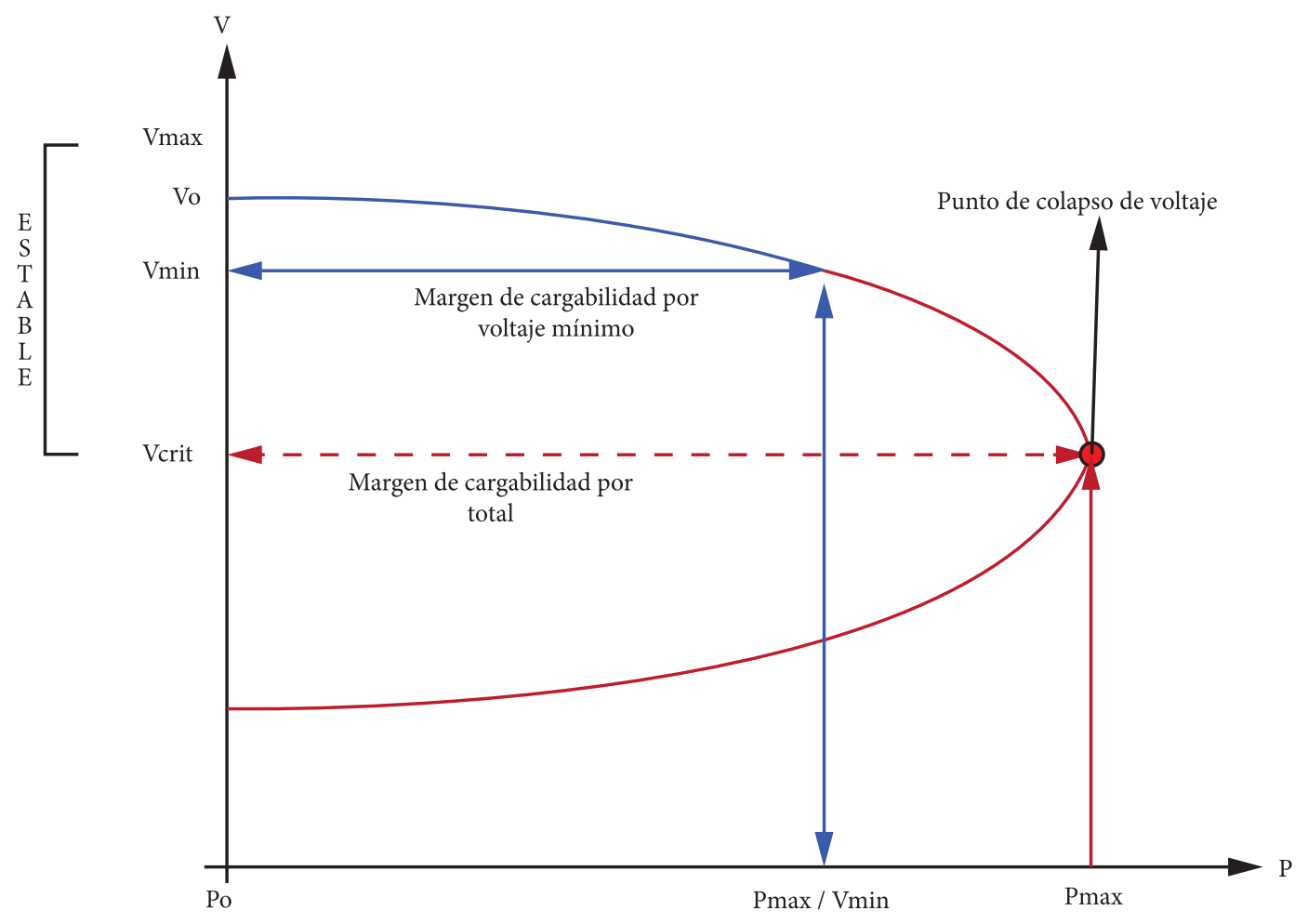


Por lo tanto,

$\Delta \mathrm{V}=J_{R}^{-1} \Delta \mathrm{VQ}$

Donde:

- $J_{R}$ es la matriz jacobiana reducida.

- $J_{R}^{-1}$ es la matriz inversa de la jacobiana reducida.

Como resultado final se obtendrá la correspondencia para el cambio en la tensión y la potencia reactiva en la cual el i-ésimo componente en la transversal de la matriz es la sensibilidad en $\mathrm{Q}-\mathrm{V}$ en el modo i, del sistema en estudio [35].

\section{Inestabilidad de voltaje en modos}

Para desarrollar la matriz diagonal de valores propios, primero se debe realizar la descomposición de la matriz jacobiana reducida, que es [36]:

$$
\begin{aligned}
& J_{R}=\Phi \Lambda \psi \\
& \Phi \psi=I ; \psi=\Phi^{-1}
\end{aligned}
$$

Donde:

- $J_{R}$ es la matriz jacobiana.

- $\Phi$ el conjunto de vectores derechos de la asociada a $\Lambda$.

- $\Lambda$ es la matriz transversal de elementos propios de $J_{R}$.

- $\psi$ el conjunto de vectores izquierdos de $J_{R}$ asociados a $\Lambda$.

Hallando la inversa de la ecuación (6), se obtiene:

$J_{R}^{-1}=\Phi \Lambda^{-1} \psi$

Despejando se tiene:

$$
\Delta V=\sum_{i} \frac{\Phi_{i} \Psi_{i}}{\lambda_{i}} \Delta \mathrm{Q}
$$

La correspondiente variación de la tensión modal es:

$$
\Delta \mathrm{v}=\Lambda^{-1} \Delta Q_{m}
$$

Entonces, tenemos:

$$
\Lambda^{-1}=\left[\begin{array}{ccc}
\lambda_{1}^{-1} & 0 & 0 \\
0 & \lambda_{2}^{-1} & 0 \\
0 & 0 & \lambda_{n}^{-1}
\end{array}\right]
$$

La i-ésima variación de voltaje modal es:

$$
\Delta V_{m i}=\lambda_{i}^{-1} \Delta Q_{m i}
$$

Donde:

- $\Delta V_{m}$ corresponde al vector del cambio de voltaje de los modos.

- $\Delta Q_{m}$ corresponde al vector del cambio de la potencia reactiva de los modos.

Con este estudio se muestra que a medida que va creciendo el valor de lambda en el modo $\mathrm{i}$ « $\lambda_{i}$ » se puede determinar la debilidad del voltaje modal, con los siguientes parámetros:

- Si el valor de « $\lambda_{i} »$ propio de la matriz jacobiana es positivo, el voltaje de la red está estable.

- Si el valor de « $\lambda_{i} »$ propio de la matriz jacobiana es cero, la red está en el límite del desequilibrio.

- Si el valor de « $\lambda_{i} »$ propio de la matriz jacobiana es negativo, la red se encuentra en un desequilibrio.

Para determinar la sensibilidad de Qv del bus $k$, que se origina en el modo i, se utiliza:

$$
\frac{\mathrm{d} V_{k}}{\mathrm{~d} Q_{k}}=\sum_{i} \frac{\Phi_{k i} \Psi_{k i}}{\lambda_{i}}
$$

\section{Factor de participación de los ramales}

Con este factor se identifica el ramal que está contribuyendo en mayor proporción a la inestabilidad de voltaje mediante la comparación de los flujos en los ramales para los estados 1 y 2 del sistema, por lo que la variación de ingreso de la potencia reactiva es, el cambio resultante es y el cambio del ángulo con respecto al modo i es [37]:

$$
\Delta Q_{m i}=J_{R}^{-1 *} J_{R}^{*} \Delta V_{m i}
$$

Se define,

$$
\Delta Q_{l \operatorname{maxi}}=\max _{j}\left(\Delta Q_{l j i}\right)
$$

Donde:

$\Delta Q_{l j i}=$ variación de pérdidas reactivas a través de la ruta de traslado $\mathrm{j}$

El elemento de la participación en los ramales es:

$$
F P R_{l j i}=\frac{\Delta Q_{l j i}}{\Delta Q_{l \max i}}
$$

\section{Factor de participación de los generadores}

Es posible identificar cuál generador está entregando más potencia reactiva a medida del cambio incremental 
Figura 3. Áreas de estabilidad e inestabilidad de la curva QV

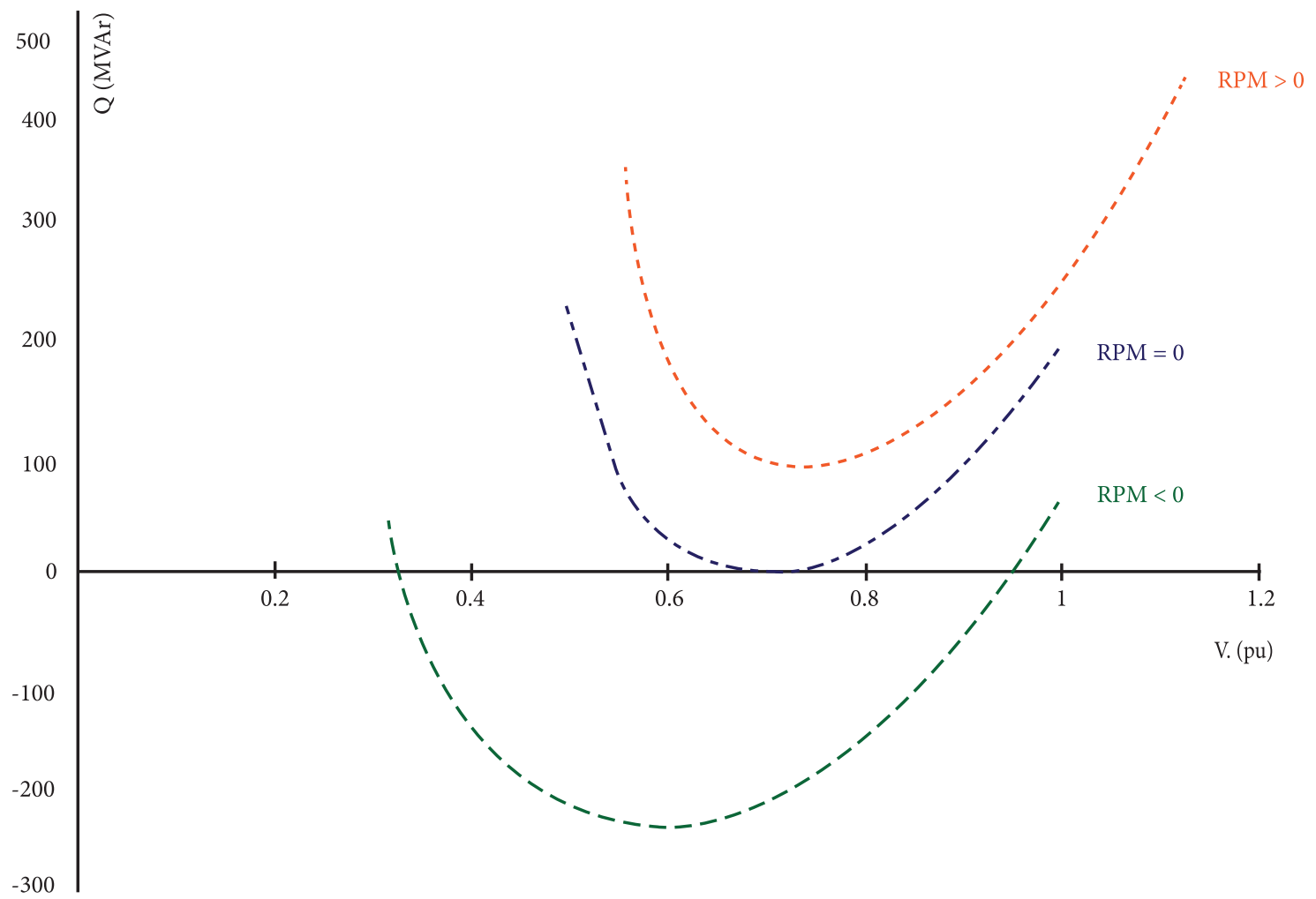

Figura 4. Curvas PV de la red eléctrica de la provincia de Manabí

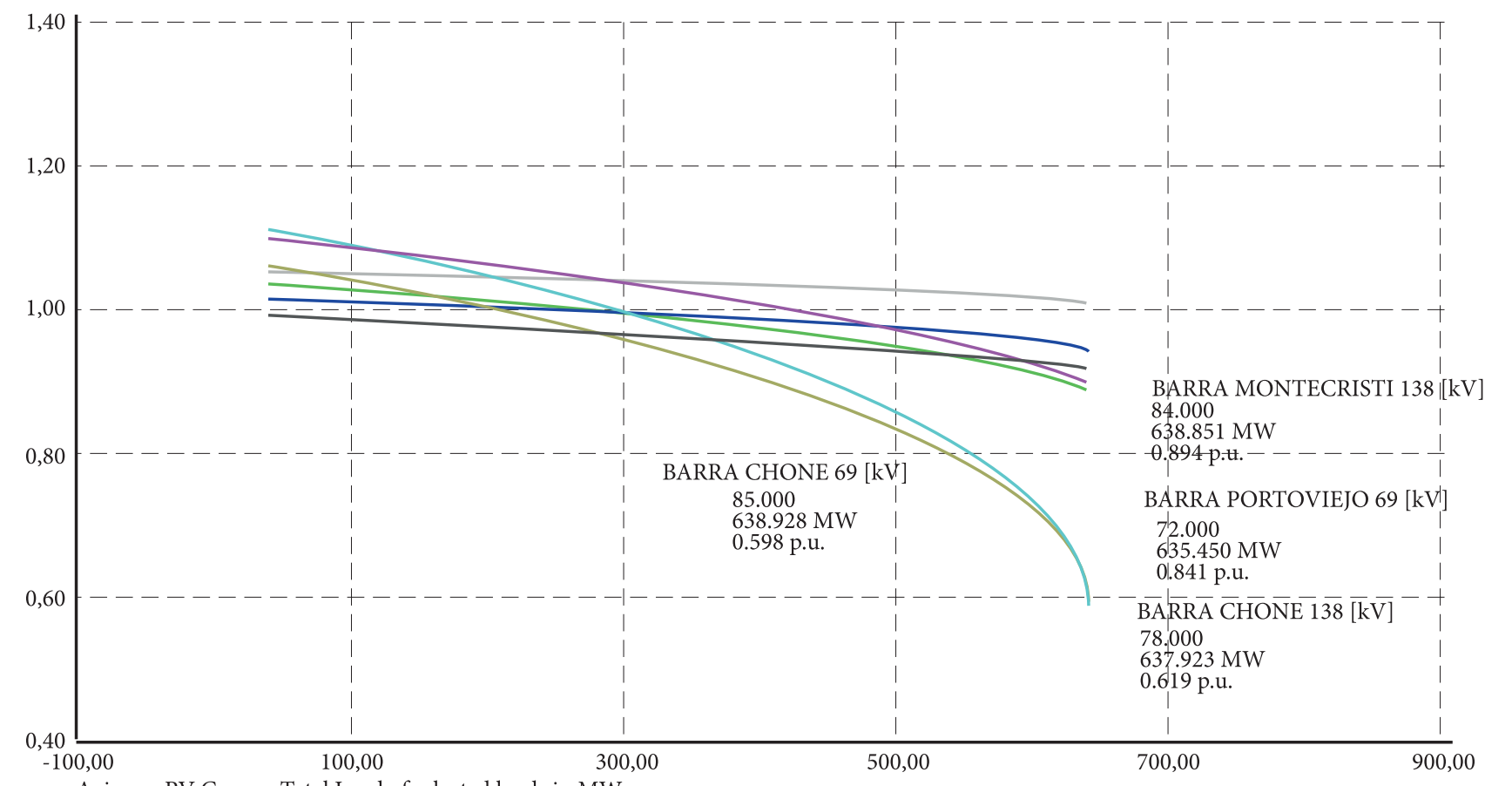

x-Axis: $\quad$ PV Curves: Total Load of selected loads in MW

BARRA PORTOVIEJO 69 [kV]: Voltage, Magnitude in p.u.

BARRA SAN GREGORIO 69 [kV]: Voltage, Magnitude in p.u.

BARRA QUEVEDO 69 [kV]: Voltage, Magnitude in p.u.

BARRA CHONE 138 [kV]: Voltage, Magnitude in p.u.

BARRA CHONE 69 [kV]: Voltage, Magnitude in p.u.

BARRA MONTECRISTI 138 [kV]: Voltage, Magnitude in p.u.

BARRA MONTECRISTI 5 [kV]: Voltage, Magnitude in p.u.

Fuente: autores. Software: DigSilent. 
Tabla 2. Resultados del FPR del sistema eléctrico de la provincia de Manabí, con una proyección de carga de 0,1 Mvar

\begin{tabular}{|c|c|c|c|c|c|c|c|}
\hline \multicolumn{2}{|c|}{ Ramales } & \multicolumn{2}{|l|}{ Estado 1} & \multicolumn{2}{|l|}{ Estado 2} & \multirow[b]{2}{*}{ Diferencia } & \multirow[b]{2}{*}{ FPR } \\
\hline From & To & $\mathrm{Q}[$ Mvar $]$ & $\mathrm{Q}[\mathbf{p . u .}$. $]$ & $\mathrm{Q}[$ Mvar $]$ & $\mathrm{Q}[\mathbf{p .} \mathbf{u}]$. & & \\
\hline 1 & 2 & $-19,971$ & $-0,199$ & 22,152 & 0,221 & 0,421 & 1 \\
\hline 1 & 4 & $-0,225$ & $-0,002$ & 0,075 & 0,007 & 0,002 & 0,006 \\
\hline 1 & 5 & 48,188 & 0,481 & 67,983 & 0,679 & 0,198 & 0,47 \\
\hline 2 & 3 & 35,724 & 0,357 & $-2,136$ & $-0,021$ & 0,378 & 0,898 \\
\hline 2 & 8 & $-5,897$ & $-0,058$ & $-33,965$ & $-0,339$ & 0,28 & 0,666 \\
\hline 5 & 6 & $-31,825$ & $-0,318$ & $-43,236$ & $-0,432$ & 0,113 & 0,27 \\
\hline 5 & 7 & 26,832 & 0,268 & 23,432 & 0,234 & 0,034 & 0,08 \\
\hline 6 & 15 & 11,131 & 0,111 & 21,848 & 0,218 & 0,107 & 0,254 \\
\hline 6 & 27 & 16,722 & 0,167 & 9,192 & 0,091 & 0,075 & 0,178 \\
\hline 8 & 9 & $-2,876$ & $-0,028$ & $-4,594$ & $-0,045$ & 0,017 & 0,04 \\
\hline 8 & 10 & $-13,248$ & $-0,132$ & $-12,796$ & $-0,127$ & 0,004 & 0,01 \\
\hline 8 & 11 & $-13,24$ & $-0,132$ & $-12,792$ & $-0,127$ & 0,004 & 0,01 \\
\hline 8 & 12 & 36,526 & 0,365 & 51,431 & 0,514 & 0,149 & 0,353 \\
\hline 8 & 27 & $-18,301$ & $-0,183$ & 0,32 & 0,003 & 0,186 & 0,442 \\
\hline 12 & 13 & $-93,186$ & $-0,931$ & $-88,555$ & $-0,885$ & 0,046 & 0,109 \\
\hline 13 & 14 & 0 & 0 & 0,325 & 0,003 & 0,003 & 0,007 \\
\hline 15 & 16 & $-29,251$ & $-0,292$ & $-28,458$ & $-0,284$ & 0,008 & 0,018 \\
\hline 15 & 19 & $-16,295$ & $-0,162$ & 0,578 & 0,005 & 0,168 & 0,4 \\
\hline 16 & 17 & 0,017 & 0,001 & $-0,915$ & $-0,009$ & 0,009 & 0,021 \\
\hline 16 & 18 & 0,015 & 0,001 & $-0,581$ & $-0,005$ & 0,005 & 0,014 \\
\hline 19 & 20 & 0 & 0 & $-1,162$ & $-0,011$ & 0,011 & 0,027 \\
\hline 19 & 21 & 0 & 0 & $-0,626$ & $-0,006$ & 0,006 & 0,014 \\
\hline 19 & 22 & $-1,684$ & $-0,016$ & $-2,672$ & $-0,026$ & 0,009 & 0,023 \\
\hline 19 & 23 & 11,033 & 0,11 & 17,532 & 0,175 & 0,065 & 0,154 \\
\hline 19 & 24 & $-25,998$ & $-0,259$ & $-26,731$ & $-0,267$ & 0,007 & 0,018 \\
\hline 23 & 25 & $-36,945$ & $-0,369$ & $-38,22$ & $-0,382$ & 0,013 & 0,029 \\
\hline 25 & 26 & 0 & 0 & 0,152 & 0,001 & 0,002 & 0,004 \\
\hline 27 & 28 & 44,696 & 0,446 & 42,685 & 0,426 & 0,02 & 0,047 \\
\hline
\end{tabular}

de la carga del sistema, si su factor de participación es más alto que otro, esto significa que un generador está aportando más potencia reactiva que la otra y con ello se puede generar una inestabilidad de voltaje por falta de compensación de potencia reactiva capacitiva, se define [38]:

$$
\Delta Q_{\text {gmaxi }}=\max _{k}\left(\Delta Q_{g k i}\right)
$$

Donde:

$\Delta Q_{g k i}=$ Cambio de la potencia reactiva para el modo $i$.

El factor de participación de generadores es:

$$
F P G_{g k i}=\frac{\Delta Q_{g k i}}{\Delta Q_{g \operatorname{maxi}}}
$$




\section{Figura 5. Curvas QV de la red eléctrica de la provincia de Manabí}

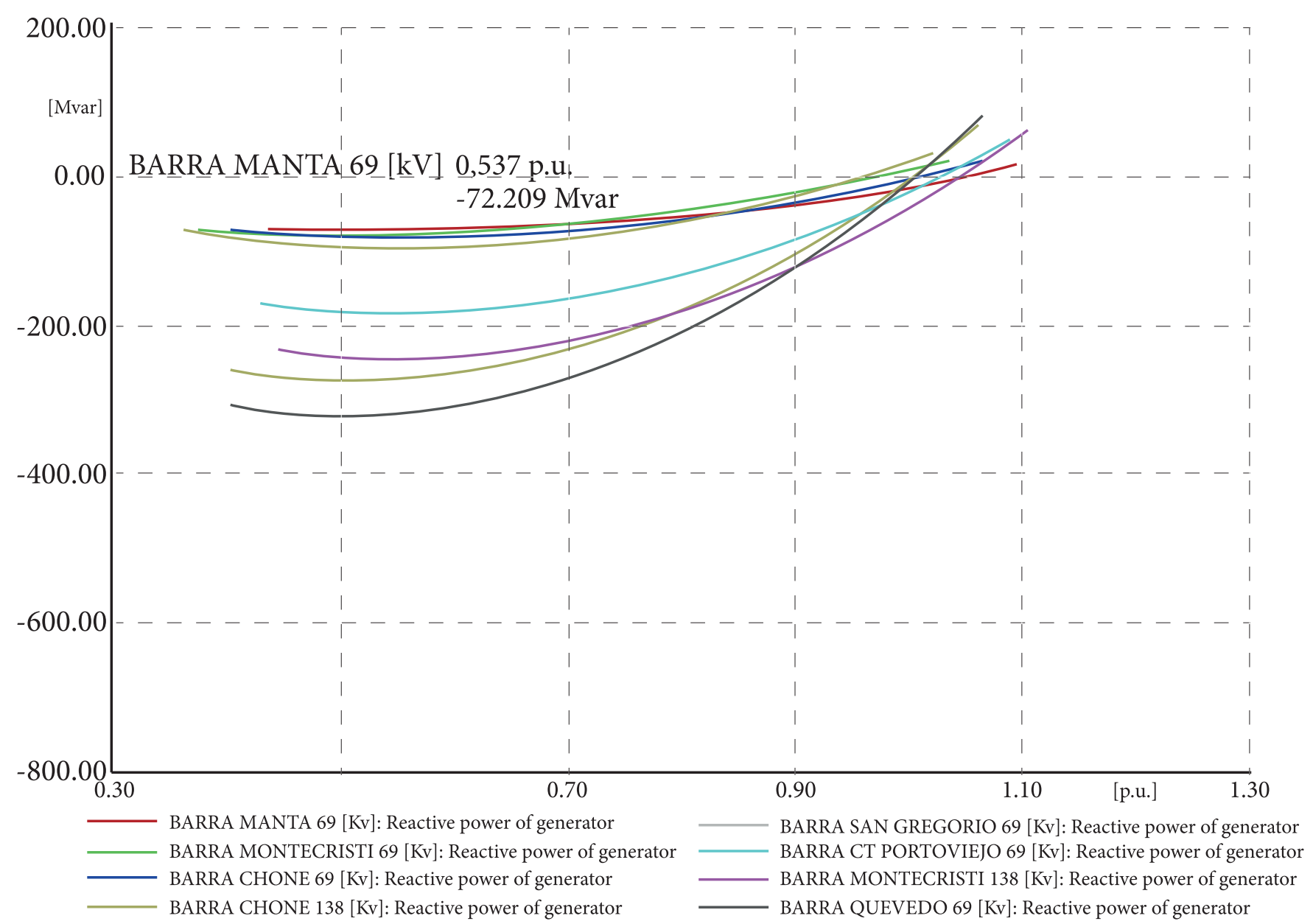

Fuente: autores. Software: DigSilent.

Los datos del sistema modelado en el DigSilent son ingresados al Matlab para realizar cálculos, utilizando algoritmos propios implementados y validados para todas las operaciones correspondientes al análisis modal. Para identificar los ramales y buses más críticos se consideran los factores de participación con valores altos [4]. Los valores de los factores de participación se obtienen simulando una perturbación de la carga de 0,1 Mvar, este valor se obtuvo por el crecimiento anual del país en el Plan Maestro de Electricidad [39].

\section{ANÁlisis y RESUltados}

\section{A. Curvas Pv}

La barra de subtransmisión más crítica es la de Chone de $69 \mathrm{kV}$, que suministra una potencia de $639.006 \mathrm{Mw}$ y 0,595 p. u. de voltaje. También la barra de Portoviejo de $69 \mathrm{kV}$ que tiene un $0,841 \mathrm{p}$. u. de voltaje que representa un suministro de potencia activa de $635.450 \mathrm{MW}$ se encuentra en una inestabilidad de voltaje. El resto de las barras se encuentran en estabilidad de voltaje ya que tienen un valor superior a 0,9 p. u. de voltaje (ver Figura 4) [23]. Este resultado se debe a que hay una excesiva demanda de potencia activa en esa barra, por lo que es necesario que exista un punto de generación cercana para compensar con su carga y estabilizar su voltaje [40].

\section{B. Curvas Qv}

La barra de subtransmisión más crítica es la de Manta de $69 \mathrm{kV}$, que tiene -72.209 Mvar y 0,537 p. u. en su voltaje. Aunque las demás cargas se encuentran en la zona de reserva de potencia reactiva, no existe un peligro de inestabilidad de voltaje por potencias reactivas inductivas (ver Figura 5).

Este resultado se debe que hay un aumento de cargas con potencias reactivas inductivas en esa barra, por lo que es necesario instalar bancos de capacitores para compensar la potencia reactiva y así estabilizar el ángulo [27].

\section{Análisis modal}

En la sección 3.4 se obtuvo los valores de los factores de participación mostrados en la tabla 2. Donde en el estado 1 se tienen las potencias reactivas en condiciones ini- 
ciales de flujo y en el estado 2 se tiene potencia reactiva con la carga aumentada de 0,1 Mvar, con ese incremento tomado del Plan Maestro de Electricidad el cual indica la proyección del ramal donde se generará una inestabilidad de voltaje, según la proyección de crecimiento del ente regulador para este sistema en análisis (ver Tabla 2).

La barra de subtransmisión más crítica es la barra 2 a 3 que es la subestación de Quevedo de $138 \mathrm{kV}$ a $69 \mathrm{kV}$, tiene un factor de participación de 0,898 p. u., que al no corregirse provocará una inestabilidad de voltaje por su excesiva carga en potencia reactiva inductiva que afecta a su ángulo del factor de potencia.

Una de las soluciones más frecuentes es la utilización de bancos de capacitores trifásicos para compensar potencias reactivas capacitivas y así mejorar el ángulo del factor de potencia.

En la tabla 3 se observan los factores de participación de los generadores, en donde el estado 1 muestra las potencias reactivas en condiciones normales de la red y en el estado 2 muestra las potencias reactivas aumentadas con 0,1 Mvar para poder hacer el análisis de las proyecciones del crecimiento de la demanda (ver Tabla 3).

La barra más crítica es la 17, que pertenece al generador 1 de Montecristi, y tiene un factor de participación de 0,346 p. u., por lo que no se considera tan grave, pero con cargas futuras puede entrar a un colapso de voltaje por excesiva demanda.

Este problema se debe a las cargas que se encuentran cerca al generador y están aumentando exponencialmente, haciendo que el generador llegue a su estado de saturación, por lo que provocaría una inestabilidad de voltaje por exceso de consumo de potencia activa [24].

Unas de las soluciones más comunes para este caso es crear fuentes de generación cercanas al generador donde se produce el colapso, para aliviar el rendimiento de la máquina y operar en porcentajes de operabilidad adecuadas.

\section{Conclusiones}

En el análisis de condiciones normales, en el DigSilent se observa que la red se encuentra operando con generadores de sobrecarga y ciertas barras con inestabilidad de voltaje.

Del análisis realizado con las curvas PV, con respecto a todas las barras que tienen sus respectivas cargas del sistema eléctrico de Manabí, se analizó que la barra más crítica es la barra de Chone de $69 \mathrm{Kv}$, ya que tiene un voltaje de 0,596 p. u., donde es necesario aumentar el sistema de generación en ese punto, puesto que llegaría a un colapso de tensión en caso de no hacerlo.

Del estudio realizado con las curvas QV, referente a todas las barras con sus respectivas cargas, se detecta que la barra más crítica por falta de compensación de potencia reactiva capacitiva es la subestación de Manta de $69 \mathrm{kV}$ ya que tiene un voltaje de 0,537 p. u., donde es necesario colocar un banco de capacitores para mejorar el ángulo y el voltaje de la red.

Cada barra es un estudio diferente, por lo que se obtienen otras curvas PV y QV, y está directamente relacionado con el dinamismo de la carga.

En el factor de participación de los ramales, la barra más crítica es la 2 a 3 que pertenece a la subestación Quevedo por lo que su FPR es de 0,898 p. u., que se debe a su incremento de potencia reactiva inductiva que afecta al ángulo de la red y es necesario poner un banco de condensadores a fin de optimizar el componente de potencia en esa red.

Referente al factor en la participación de los generadores, la barra más crítica es la 17 que es del generador 1 de Montecristi que tiene un FPG de 0,346 p. u., por lo que la red estaría pronto a entrar en una inestabilidad de voltaje por su máxima carga al generador, debido a lo cual es preciso incrementar la facultad de generación ya que existe una gran demanda.

\section{REFERENCIAS}

[1] P. D. E. Distribución, «Voltage stability analysis in power distribution feeder reconfiguration», vol. 33, N. $^{\circ} 41$, pp. 30-35, 2009.

[2] Arconel 006/2020, «Resolución N. . Arconel-006/2020», pp. 1-58, 2020, [Online]. Available: http://www. cna-ecuador.com/wp-content/uploads/2020/06/RegSust-Reg-ARCONELOO1-20-Directorio-res-006-20-firm. pdf

[3] Arconel, «Resolución N. ${ }^{\circ}$ Arconel-053/18», Arconel, pp. 1-40, 2018.

[4] «PME 2016-2025.pdf», Minist. Electr. y Energía Renov., p. $439,2017$.

[5] B. Brusiłowicz, W. Rebizant and J. Szafran, «A new method of voltage stability margin estimation based on local measurements», APAP 2011 - Proc. 2011 Int. Conf. Adv. Power Syst. Autom. Prot., vol. 3, pp. 2443-2447, 2011, DOI: 10.1109/APAP.2011.6180655.

[6] M. A. Joodi, I. K. Ibraheem, and F. M. Tuaimah, «Power transmission system midpoint voltage fixation using SVC with genetic tuned simple PID controller», Int. J. Eng. Technol., vol. 7, N. ${ }^{\circ}$ 4, pp. 5438-5443, 2018, Dor: 10.14419/ ijet.v7i4.24799.

[7] G. Chen, J. L. Moiola, and H. O. Wang, «Bifurcation control: Theories, methods, and applications», Int. J. Bifurcat. Chaos, vol. 10, N. ${ }^{\circ} 3$, pp. 511-548, 2000, DoI: 10.1142/S0218127400000360.

[8] Z. Wang, X. Yin, Y. Chen, J. Lai, L. Li, and Z. Qi, «DstATсом integrated with Y-y connection transformer for reactive power compensation», Int. J. Electr. Power Energy Syst., vol. 117, ‥ ${ }^{\circ}$ september 2018, p. 105721, 2020, DOI: 10.1016/j.ijepes.2019.105721.

[9] T. Cui, Y. Shen, Z. Hu, J. Song, H. Guo and X. Wang, 
«Voltage regulation of synchronous condensers and switching capacitors in power grids with a UHV DC/AC System», Proc. - 2018 3rd Int. Conf. Smart City Syst. Eng. ICSCSE 2018, N. ${ }^{\circ} 1$, pp. 552-556, 2018, DOI: 10.1109/ICSCSE.2018.00118.

[10] V. F. Pires, A. V. Pombo and J. M. Lourenço, «Multi-objective optimization with post-pareto optimality analysis for the integration of storage systems with reactive-power compensation in distribution networks», J. Energy Storage, vol. 24, N. ${ }^{\circ}$ april, p. 100769, 2019, doi: 10.1016/j.est.2019.100769.

[11] M. Hasani and M. Parniani, «Method of combined static and dynamic analysis of voltage collapse in voltage stability assessment», Proc. IEEE Power Eng. Soc. Transm. Distrib. Conf., vol. 2005, pp. 1-6, 2005, DOI: 10.1109/ TDC.2005.1547182.

[12] M. S. Saddique et al., «Solution to optimal reactive power dispatch in transmission system using meta-heuristic techniques-Status and technological review», Electr. Power Syst. Res., vol. 178, N. ${ }^{\circ}$ august 2019, p. 106031, 2020, Dor: 10.1016/j.epsr.2019.106031.

[13] B. Singh and R. Kumar, "A comprehensive survey on enhancement of system performances by using different types of FACTS controllers in power systems with static and realistic load models», Energy Reports, vol. 6, pp. 55-79, 2020, DoI: 10.1016/j.egyr.2019.08.045.

[14] S. Chansareewittaya, "Optimal allocations of FACTs controllers for economic dispatch using evolutionary programming», ICSEC 2017 - 21st Int. Comput. Sci. Eng. Conf. 2017, Proceeding, vol. 6, pp. 1-4, 2018, DoI: 10.1109/ICSEC.2017.8443901.

[15] M. Tituaña and D. Carrión, «Análisis de inestabilidad de tensión en el sistema eléctrico de potencia usando el análisis modal», p. 22, 2016.

[16] P. Cao et al., «Analysis of electrical length compensation types for tuned half-wavelength transmission lines», Int. J. Electr. Power Energy Syst., vol. 115, N. ${ }^{\circ}$ August 2019, p. 105520, 2020, DoI: 10.1016/j.ijepes.2019.105520.

[17] P. A. Löf, T. Smed, G. Andersson and D. J. Hill, "Fast calculation of a voltage stability index», IEEE Trans. Power Syst., vol. 7, N. ${ }^{\circ}$ 1, pp. 54-64, 1992, DOI: 10.1109/59.141687.

[18] M. Miranda, «Diseño de sistema de generación fotovoltaica para viviendas conectadas a la red de distribución, en el contexto de la Ley N. ${ }^{\circ} 20.571 », 2016$.

[19] Á. A. Recalde, «Estabilidad de los sistemas de potencia: problemáticas en escenarios complejos», Research Gate, vol. 1, N. ${ }^{\circ}$ october 2014, p. 6, 2015, [Online]. Available: https://www.researchgate.net/profile/Angel_ Recalde/publication/273450101_ESTABILIDAD_DE_LOS_ SISTEMAS_DE_POTENCIA_PROBLEMATICAS_EN_ESCENARIOS_COMPLEJOS/links/550204b60cf2d60c0e62981f/ ESTABILIDAD-DE-LOS-SISTEMAS-DE-POTENCIA-PROBLEMATICAS-EN-ESCENARIOS-COMPL

[20] A. Amer, «Voltage collapse prediction for interconnected power systems», Morgantown, West Virginia, 2000, [Online]. Available: http://www.wvuscholar.wvu.edu:8881/ exlibris/dtl/d3_1/apache_media/L2v4bGlicmlzL2R0bc9kM18xL2Fwrwnozv9tzwrpys80otg5.pdf

[21] N. Manjul and M. S. Rawat, «Pv/Qv Curve based optimal placement of static var system in power network using DigSilent power factory», 8th IEEE Power India Int. Conf. PIICON 2018, pp. 1-6, 2018, DOI: 10.1109/POWERI.2018.8704441.

[22] A. C. Zambroni De Souza, F. W. Mohn, I. F. Borges and T. R. Ocariz, «Using PV and QV curves with the meaning of static contingency screening and planning», Electr. Power Syst. Res., vol. 81, N. ${ }^{\circ}$ 7, pp. 1491-1498, 2011, DOI: 10.1016/j.epsr.2011.02.012.

[23] H. G. P. Kesse, «C=min]», N..$^{\circ}$ july, p. 9935, 1986.

[24] M. A. Ríos, A. Torres M., and M. T. de Torres, «Estabilidad de voltaje en sistemas de potencia: Caso de aplicación a la EEB», Rev. Ing., N. ${ }^{\circ}$ 8, pp. 19-24, 1997, DOI: 10.16924/revinge.8.4.

[25] V. N. Sewdien, R. Preece, J. L. R. Torres, and M. A. M. M. Van Der Meijden, «Evaluation of PV and QV based voltage stability analyses in converter dominated power systems» Asia-Pacific Power Energy Eng. Conf. APPEEC, vol. 2018-Octob, pp. 161-165, 2018, DOI: 10.1109/ APPEEC.2018.8566421.

[26] R. S. Moura, A. C. Zambroni de Souza, B. I. Lima Lopes, and F. W. Mohn, «Effects of QV curves in the dynamic behaviour of power systems» IET Gener. Transm. Distrib., vol. 10, N. ${ }^{\circ} 12$, pp. 2861-2870, 2016, DoI: 10.1049/ iet-gtd.2015.1042.

[27] A. Bonini Neto and D. Amancio Alves, «Singularities analysis of the jacobian matrix modified in the continuation power flow: performance evaluation», IEEE Lat. Am. Trans., vol. 15, N. ${ }^{\circ} 11$, pp. 2137-2143, 2017, DoI: 10.1109 / TLA.2017.8070419.

[28] K. Vu, M. M. Begovic, D. Novosel and M. M. Saha, «Use of local measurements to estimate voltage-stability margin», IEEE Power Ind. Comput. Appl. Conf., pp. 318-323, 1997.

[29] D. Eltigani, «Method of computing maximum loadability, using continuation power flow», pp. 0-4, 2013.

[30] N. C. Yang and H. C. Chen, «Decomposed Newton algorithm-based three-phase power-flow for unbalanced radial distribution networks with distributed energy resources and electric vehicle demands», Int. J. Electr. Power Energy Syst., vol. 96, . . . september 2017, pp. 473483, 2018, Dor: 10.1016/j.ijepes.2017.09.042.

[31] D. Hau and A. Lee, «Equivalent nodal analysis», vol. 31, N. ${ }^{\circ}$ 1, pp. 454-463, 2016.

[32] T. Van Cutsem, «Voltage instability: phenomena, countermeasures, and analysis methods» Proc. IEEE, vol. 88, N. ${ }^{\circ}$ 2, pp. 208-227, 2000, DoI: 10.1109/5.823999.

[33] Y. Song, D. J. Hill, and T. Liu, «State-in-mode analysis of the power flow jacobian for static voltage stability», Int. J. Electr. Power Energy Syst., vol. 105, no. September 2018, pp. 671-678, 2019, DOI: 10.1016/j.ijepes.2018.09.012. 